\title{
UTILIDAD DE LA PUNTUACIÓN DE BOEY Y PORTSMOUTH-POSSUM FISIOLÓGICO EN PACIENTES CON ÚLCERA PÉPTICA PERFORADA*
}

\author{
Drs. Marcelo A. Beltrán S. ${ }^{1,2}$, Ramón I. Díaz J. ${ }^{1}$, Catalina Valenzuela V. ${ }^{1}$, \\ Gabriel Iribarren R. ${ }^{2}$, Andrea De Balanzo A. ${ }^{2}$, Francisco Rodríguez V. ${ }^{1,2}$ \\ 1 Servicio de Cirugía, Hospital de La Serena. \\ 2 Departamento de Clínicas, Facultad de Medicina, Universidad Católica del Norte. La Serena. \\ Chile.
}

\begin{abstract}
Performance of Boey score and physiologic Portsmouth-POSSUM in patients with perforated peptic ulcer

Introduction: Surgical scores such as Boey and physiologic Portsmouth-POSSUM have been independently applied to patients with perforated ulcer to stratify their surgical risk. However, there are no studies comparing both scores. The purpose of this study was to compare the performance of Boey score and Portsmouth-POSSUM in patients with perforated peptic ulcer. Methods: A retrospective comparative study was performed including 108 consecutive patients older than 15 -years submitted to emergency surgery from January 2002 to June 2012. Patients operated on for perforated gastric cancer were excluded. The primary outcome measure was to compare the performance of Portsmouth-POSSUM and Boey score. Secondary outcome measures were to determine cutoff points for Portsmouth-POSSUM, Boey score, C-reactive protein (CRP), and white blood cells (WBC) count, to predict patients at risk for complications. Results: The best cutoff point for CRP was $37.5 \mathrm{mg} / 1$, and for WBC was $11.600 \mathrm{~mm}^{3}$ (OR 2.9 and 4.4). The best cutoff point for physiologic Portsmouth-POSSUM was 14, for surgical Portsmouth-POSSUM were 12, and for predictive Portsmouth-POSSUM was $0.8 \%$. A time of perforation higher than $24 \mathrm{~h}$ had an OR of 35, and Boey score of $3 \mathrm{had}$ an OR of 38.3. When Boey score was 2, with preoperative shock and time of perforation higher than $24 \mathrm{~h}$ being the positive variables, the OR was 194.3. Conclusions: Boey score performed better than Portsmouth-POSSUM, remaining a more specific score to stratify patients submitted to emergency surgery for perforated peptic ulcer.
\end{abstract}

Key words: Perforated peptic ulcer, Boey score, Portsmouth-POSSUM.

\section{Resumen}

Introducción: Puntuaciones pronósticas como la de Boey y el Portsmouth-POSSUM han sido utilizadas en pacientes con úlcera perforada para estratificar el riesgo quirúrgico. No existen estudios que comparen am-

\footnotetext{
Los autores declaran no tener conflicto de interés.

Correspondencia: Dr. Marcelo A. Beltrán S.

beltran_01@yahoo.com
}

*Recibido el 2 de enero de 2014 y aceptado para publicación el 3 de abril de 2014. 
bas puntuaciones. El objetivo del presente estudio es el de comparar el rendimiento de estas puntuaciones en pacientes con úlcera péptica perforada. Métodos: Se diseñó un estudio comparativo retrospectivo que incluyó 108 pacientes consecutivos mayores de 15 años sometidos a cirugía de urgencia entre enero de 2002 y junio de 2012. Se excluyeron pacientes operados por cáncer gástrico perforado. El objetivo principal fue comparar el rendimiento de la puntuación de Boey con Portsmouth-POSSUM. Los objetivos secundarios fueron determinar los puntos de corte para Portsmouth-POSSUM, puntuación de Boey, proteína C-reactiva (PCR) y recuento de leucocitos (RL) como factores predictivos de riesgo. Resultados: El mejor punto de corte para PCR fue $37,5 \mathrm{mg} / \mathrm{l}$ y para RL $11.600 \mathrm{~mm}^{3}$ (OR 2,9 y 4,4). El mejor punto de corte para Portsmouth-POSSUM fisiológico fue 14, para Portsmouth-POSSUM quirúrgico fue 12 y para Portsmouth-POSSUM predictivo fue $0,8 \%$. Un tiempo de perforación mayor a 24 h tenía un OR de 35 y un puntaje de Boey de 3 tenía un OR de 38,3. Cuando el puntaje de Boey fue 2 con las variables choque preoperatorio y perforación mayor a $24 \mathrm{~h}$, el OR fue 194,3. Conclusiones: La puntuación de Boey presentó mejor rendimiento que Portsmouth-POSSUM, representando una puntuación más específica para estratificar pacientes sometidos a cirugía de urgencia por úlcera perforada.

Palabras clave: Úlcera péptica perforada, puntuación de Boey, Portsmouth-POSSUM.

\section{Introducción}

La incidencia de cirugía de urgencia para úlcera péptica perforada (UPP) permanece estable asociándose a morbilidad y mortalidad elevadas debido a la presentación tardía y al choque preoperatorio como factores principales ${ }^{1-8}$. Así como en otras patologías quirúrgicas, se han realizado intentos para cuantificar el riesgo quirúrgico. Para este objetivo, la puntuación más utilizada en UPP es la desarrollada por John Boey ${ }^{2,9-12}$. Esta puntuación asigna un valor de 1 a factores de riesgo independientes: patología asociada severa, choque preoperatorio y perforación mayor a $24 \mathrm{~h}$. La aplicación práctica se demostró mediante el aumento progresivo de la mortalidad con un mayor número de factores ${ }^{11}$. La puntuación de Boey (P-Boey) es considerada como el estándar de oro para la evaluación de pacientes sometidos a operaciones de urgencia por UPP ${ }^{13-18}$. Recientemente la parte fisiológica del Physiological and Operative Severity Score for the enUmeration of Mortality and Morbidity score (Portsmouth-POSSUM) ha sido aplicada a pacientes operados por UPP ${ }^{19}$. El Portsmouth-POSSUM (P-POSSUM) evalúa el estado fisiológico preoperatorio y postoperatorio del paciente midiendo las patologías asociadas, parámetros de laboratorio y las características de la cirugía. El resultado es una puntuación compuesta por dos partes, una fisiológica preoperatoria y otra quirúrgica postoperatoria, las que proveen una tasa de morbilidad y mortalidad predictiva ${ }^{20,21}$. Las puntuaciones de Boey y P-POSSUM fisiológico deben aplicarse en el preoperatorio, por otro lado el P-POSSUM quirúrgico se aplica después de la operación. No existen estudios comparando P-Boey con P-POSSUM en pacientes con UPP. Debido a que las alteraciones fisiológicas y las patologías asociadas, principalmente respiratorias, cardiacas o metabólicas, constituyen factores de riesgo independientes para morbilidad y mortalidad postoperatoria ${ }^{6-8,15}$, es plausible pensar que P-POSSUM tendrá un mejor rendimiento que P-Boey. El objetivo de este estudio es comparar el rendimiento de P-Boey y P-POSSUM en pacientes operados por UPP.

\section{Métodos}

Se diseñó un estudio retrospectivo, descriptivo no-intervencionista que incluyó 108 pacientes consecutivos sometidos a cirugía de urgencia por UPP en nuestra institución entre enero de 2002 y junio de 2012. Se incluyeron todos los pacientes operados mayores de 15 años. Se excluyeron pacientes operados por cáncer gástrico perforado.

\section{Variables}

Se midieron variables clínicas, de laboratorio y quirúrgicas. El recuento de leucocitos (RL) y la proteína $\mathrm{C}$ reactiva (PCR) fueron analizados como factores pronósticos independientes, los valores normales en nuestra institución para RL son de 4.000 a $11.300 \mathrm{~mm}^{3}$ y para PCR de 0 a $5 \mathrm{mg} / 1$. Se midieron las variables necesarias para calcular P-Boey y PPOSSUM de acuerdo a las definiciones establecidas $^{9-12,20,21}$. El tiempo de perforación se definió como el período de tiempo entre el inicio de los síntomas y la operación. Las complicaciones se describen de acuerdo a la clasificación de Clavien ${ }^{22}$.

\section{Objetivos principales}

El objetivo primario principal fue comparar el rendimiento de P-POSSUM y P-Boey. Los objetivos secundarios fueron determinar los puntos de corte con la mejor sensibilidad y especificidad para P-POSSUM, P-Boey, PCR y RL para identificar pacientes de riesgo. 


\section{Estadística}

El análisis fue realizado con el programa SPSS 11,0. Se utilizó estadística descriptiva para el reporte de los resultados y estos se analizaron con el test $\chi^{2}$ y el test no-paramétrico Kolmogorov-Smirnov. Los resultados de P-POSSUM y P-Boey fueron analizados mediante un modelo de discriminación para distinguir pacientes que experimentaron complicaciones de los que no lo hicieron. El modelo de discriminación fue medido utilizando curvas Receiver-Operator Characteristics (ROC). En las curvas ROC el Área Bajo de la Curva (ABC) representa la probabilidad de que los pacientes que sufrieron complicaciones tuvieran una mayor probabilidad de sufrir complicaciones que los pacientes que no sufrieron complicaciones. Un ABC de 0,5 indica que el modelo no predice mejor que la casualidad. El modelo es excelente cuando el $\mathrm{ABC}$ es 1,0 . Si el $\mathrm{ABC}$ es de 0,8 a 0,9 se considera muy bueno. Cuando el $\mathrm{ABC}$ es 0,7 a 0,8 se considera bueno. $\mathrm{Si}$ el $\mathrm{ABC}$ es 0,6 a 0,7 se considera razonable y cuando es 0,5 o menor es considerado pobre ${ }^{23,24}$. Los puntos de corte se calcularon desde las tablas de sensibilidad y especificidad de las curvas ROC y se reportan con intervalos de confianza de 95\% (IC 95\%). Se calcularon los Odds Ratio (OR) para evaluar la probabilidad de que los factores con los mejores puntos de corte se asociaran al evento.

\section{Resultados}

\section{Características generales}

La serie se compuso principalmente de pacientes masculinos (104 casos, 96\%, p <0,0001), la mayoría de ellos menores a 60 años (87 pacientes, $80,5 \%$ ). La edad promedio fue 43,7 $\pm 14,6$ años (18-85). El promedio de tiempo desde el inicio de los síntomas hasta la operación fue $22,1 \pm 29,8 \mathrm{~h}$ (2-137), la mayoría de los pacientes se presentaron entre 4 y $12 \mathrm{~h}$ (67 pacientes, 62\%, p < 0,0001), de ellos 83 pacientes $(77 \%, \mathrm{p}<0,0001)$ llegaron dentro de las primeras $24 \mathrm{~h}$. Cuatro pacientes se presentaron entre las 25 y $48 \mathrm{~h}$ y 21 pacientes (19\%) llegaron después de $49 \mathrm{~h}$. La mayor parte de los pacientes no tenían patologías asociadas (76 casos, $70 \%, \mathrm{p}<0,0001)$. Las patologías más frecuentes fueron la diabetes y la hipertensión arterial (25 pacientes, 24\%). Frecuentemente los pacientes consumían alcohol y fumaban 5 o más cigarrillos diarios (Tabla 1).

\section{RL y PCR}

El promedio de RL fue $11.745 \pm 2.764 \mathrm{~mm}^{3}$ (7.900-21.500), la mayoría de los pacientes tenían un RL mayor a $11.500 \mathrm{~mm}^{3}$ (93 casos, $86 \%$, $\mathrm{p}<0,0001)$. El promedio de la PCR fue $118,2 \pm$
$122 \mathrm{mg} / \mathrm{l}(0,2-435)$, la mayoría de los pacientes tenían una PCR mayor a $6,3 \mathrm{mg} / 1$ (94 casos, $87 \%$, $\mathrm{p}<0,0001)$.

\section{Puntuación de Boey}

La mayoría de los pacientes tenían un P-Boey de 0 (55 casos, $51 \%, \mathrm{p}<0,0001) ; 37$ pacientes $(34 \%)$ tenían 1 punto; 13 pacientes (12\%) tenían 2 puntos; y 3 pacientes tenían 3 puntos.

\section{Portsmouth-POSSUM}

El P-POSSUM fisiológico tenía un puntaje promedio de 16,5 $\pm 3,8$ (12-29), la mayoría de los pacientes tenían entre 12 y 18 puntos ( 83 casos, $77 \%$, $\mathrm{p}<0,0001)$. El P-POSSUM quirúrgico tenía un promedio de 13,4 $\pm 1,6$ puntos (10-19), la mayoría de los pacientes tenían entre 13 y 14 puntos ( 77 casos, $71 \%, \mathrm{p}<0,0001)$. El promedio del P-POSSUM predictivo fue $2,2 \pm 2,8 \%(0,5-22)$, la mayoría de los pacientes tuvieron un valor de $0,6 \%$ a $1,3 \%$ (68 casos, $63 \%, \mathrm{p}<0,0001)$.

\section{Procedimientos quirúrgicos}

La mayoría de los pacientes fueron resueltos mediante sutura simple de la úlcera (Tabla 2). El promedio de tiempo quirúrgico fue $61,6 \pm 29,7 \mathrm{~min}$ (25-180), en la mayoría de los casos la cirugía duró entre 60 y $90 \min (97$ pacientes, $90 \%, \mathrm{p}<0,0001)$.

\section{Tiempo de hospitalización y complicaciones}

El promedio de estadía hospitalaria fue 9,3 $\pm 8,7$ días (2-76), la mayoría de los pacientes estuvo hos-

Tabla 1.

\begin{tabular}{|lcc|}
\hline Patología asociada & n (\%) & p \\
Ninguna & $76(70)$ & $<0,0001$ \\
Diabetes/hipertensión arterial & $13(12)$ & 0,296 \\
Diabetes & $6(6)$ & 0,845 \\
Hipertensión arterial & $6(6)$ & 0,846 \\
Patología coronaria & $1(1)$ & 0,997 \\
Consumo de alcohol & $87(81)$ & $<0,0001$ \\
Tabaquismo & $85(79)$ & $<0,0001$ \\
Sintomatología previa de úlcera & $37(34)$ & 0,002 \\
Infección conocida por & $6(6)$ & $<0,0001$ \\
Helicobacter Pylori & & \\
\hline
\end{tabular}

La mayoría de los pacientes no tenían patologías asociadas $(\mathrm{p}<0,0001)$. Las patologías asociadas no influenciaron los resultados. El consumo de alcohol y cigarrillos fue común $(\mathrm{p}<0,0001)$. Muy pocos pacientes tenían patología ulcerosa previa conocida o infección por Helicobacter Pylori. 
Tabla 2.

\begin{tabular}{|lcc|}
\hline Procedimientos quirúrgicos & n (\%) & p \\
Sutura simple & $71(66)$ & $<0,0001$ \\
Sutura + biopsia & $25(23)$ & \\
Parche de Graham & $9(8)$ & \\
Sutura + esplenectomía & $3(3)$ & \\
\hline
\end{tabular}

El procedimiento habitualmente realizado fue la sutura simple de la úlcera. Otros procedimientos fueron realizados de acuerdo a la preferencia del cirujano tratante.

Tabla 3.

\begin{tabular}{|llrr|}
\hline Clavien & Complicación & n & $(\%)$ \\
I & Infección de sitio quirúrgico superficial & 13 & $(13)$ \\
& Ileo prolongado & 2 & $(2)$ \\
& Infección urinaria & 2 & $(2)$ \\
& Total & 17 & $(16)$ \\
II & Neumonía postoperatoria & 14 & $(13)$ \\
& Trombosis venosa profunda & 7 & $(7)$ \\
& Total & 21 & $(20)$ \\
IIIb & Evisceración postoperatoria & 8 & $(8)$ \\
& Fístula gastro-cutánea & 1 & $(1)$ \\
& Total & 9 & $(9)$ \\
IVa & Tromboembolismo pulmonar & 6 & $(6)$ \\
Complicaciones & Hernia incisional & 8 & $(8)$ \\
tardías & Cuerpo extraño & 1 & $(1)$ \\
\hline
\end{tabular}

De acuerdo con la clasificación de Clavien, la mayoría de los pacientes complicados sufrieron complicaciones menores. Las complicaciones tardías se presentaron 3 o más meses después de la cirugía. pitalizado entre 4 a 10 días (84 casos, 78\%, $\mathrm{p}<0,0001)$. Las estadías más prolongadas se asociaron a pacientes que sufrieron complicaciones $(\mathrm{p}<0,0001)$. La mayor parte de los pacientes (85 casos, 79\%, p $<0,0001$ ) no presentó complicaciones. La mayoría de las complicaciones fueron menores (Tabla $3)$. En la serie no existió mortalidad postoperatoria.

\section{Rendimiento de la PCR y RL}

El mejor punto de corte para la PCR fue $37,5 \mathrm{mg} / \mathrm{l}$ (Figura 1A; ABC 0,7; $\mathrm{p}=0,002$; IC $95 \% 0,577 / 0,848)$ y para $R L$ fue $>11.600$ $\mathrm{mm}^{3}$ (Figura 1B; ABC 0,8; $<<0,0001$; IC $95 \%$ 0,676/0,871). Ambos parámetros se asociaron al evento (OR 2,9 y 4,4).

\section{Comparaciones entre P-POSSUM y Boey}

La puntuación de Boey se comparó con P-POSSUM mediante el uso de curvas ROC. El mejor punto de corte para P-POSSUM fisiológico fue 14 (Figura 2A; ABC 0,7; p = 0,013; IC 95\% 0,515/0,824; OR 3,6). Para P-POSSUM quirúrgico fue 12 (Figura 2B; ABC 0,8; $<<0,0001$; IC 95\% 0,667/0,893; OR 6,2). Para P-POSSUM predictivo fue (Figura 2C; ABC 0,8; p < 0,0001; IC 95\% $0,649 / 0,908$; OR 4,6). Estos resultados fueron comparables a un P-Boey mayor a 1 (Figura 3; $\mathrm{ABC} 0,8 ; \mathrm{p}<0,0001$; IC 95\%

\section{A. Proteína $\mathrm{C}$ reactiva}

ROC Curve

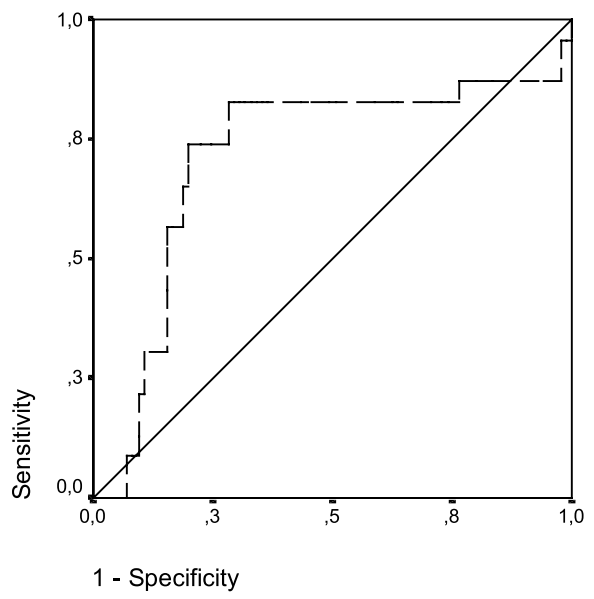

B. Recuento de leucocitos ROC Curve

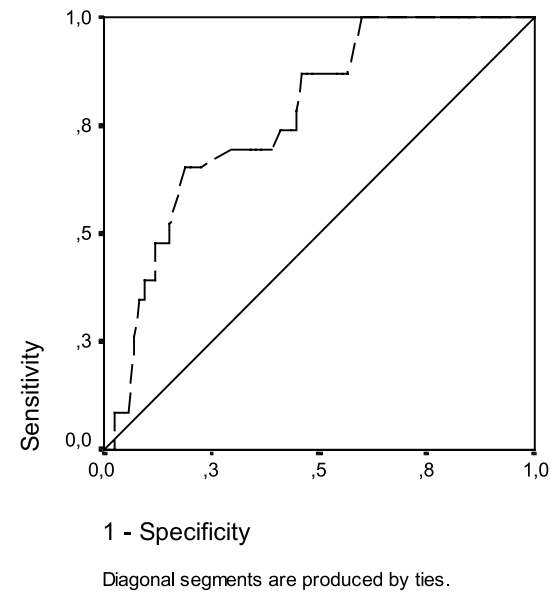

Figura 1. El ABC para la PCR $(0,7)$ se considera razonable y el ABC para RL $(0,8)$ se considera bueno de acuerdo al modelo de discriminación utilizado. 


\section{A. P-POSSUM fisiológico}

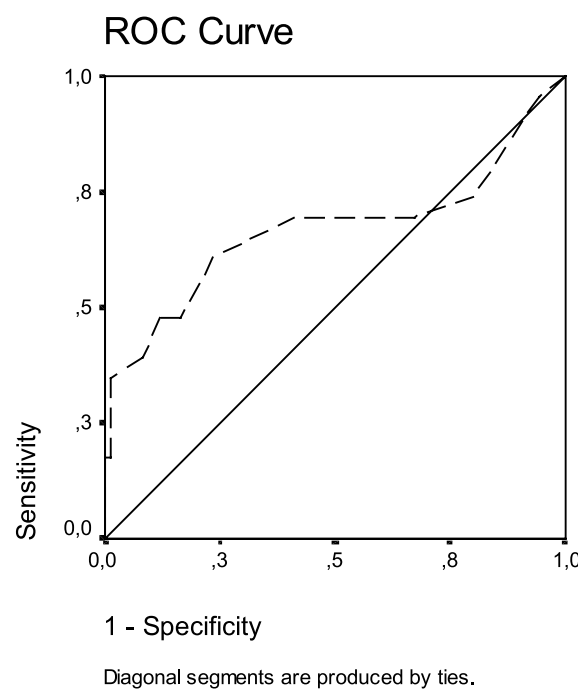

\section{B. P-POSSUM quirúrgico} ROC Curve

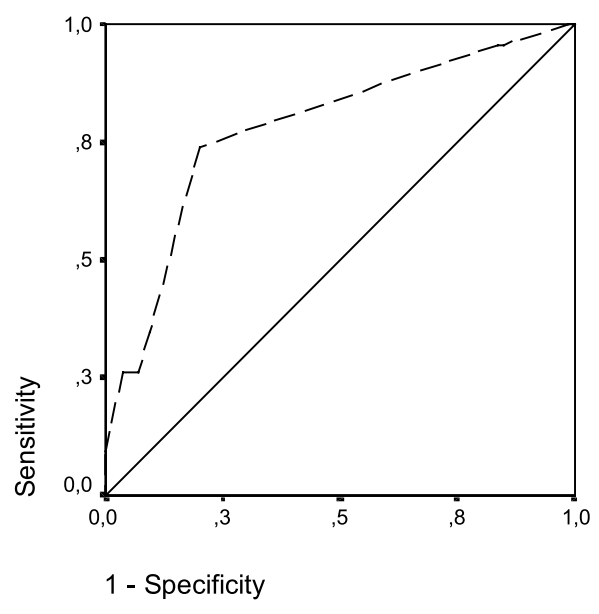

Diagonal segments are produced by ties.

\section{P-POSSUM predictivo}

\section{ROC Curve}

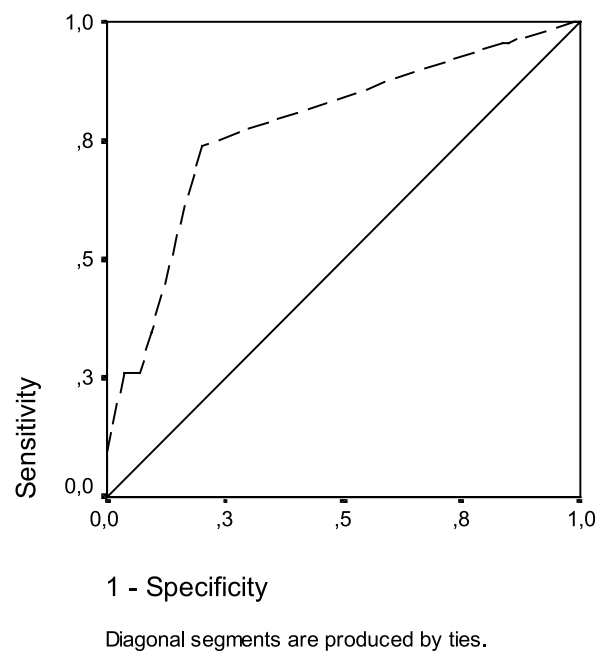

Figura 2. Las mejores ABC fueron para P-POSSUM quirúrgico y predictivo, el P-POSSUM fisiológico tuvo un ABC de 0,7 .

0,700/0,928; OR 5,7). Adicionalmente, todas las variables de P-Boey fueron evaluadas individualmente y en todas sus posibles combinaciones. Un tiempo de perforación mayor a $24 \mathrm{~h}$ tuvo una excelente $\mathrm{ABC} 1,0 ;$ OR 35 . Una puntuación mayor a 3 también presentó una excelente ABC 1,0; OR 38,3). Cuando P-Boey fue 2 con las variables choque preoperatorio y tiempo de perforación mayor a 24 h, el ABC fue bueno $(0,8)$ y los OR fueron 194,3 (Tabla 4). 
Tabla 4.

\begin{tabular}{|lcccc|}
\hline Puntuación de Boey & ABC & OR & p & IC 95\% \\
0 & 0,3 & 0,003 & 0,010 & $0,252 / 0,462$ \\
Boey-1a: Patología asociada & 0,5 & 0,5 & 0,511 & $0,348 / 0,575$ \\
Boey-1b: Choque preoperatorio & 0,8 & 0,3 & $<0,0001$ & $0,677 / 0,956$ \\
Boey-1c: Perforación > 24 h & 1,0 & 35 & 0,017 & $0,826 / 0,984$ \\
Boey-1a + Boey-1b = Boey 2ab & 0,8 & 0,1 & 0,020 & $0,600 / 1,016$ \\
Boey-1a + Boey-1c = Boey 2ac & 0,7 & 3,4 & 0,024 & $0,635 / 0,964$ \\
Boey-1b + Boey-1c = Boey 2bc & 0,8 & 194,3 & 0,006 & $0,610 / 0,970$ \\
Boey-1a + Boey-1b + Boey-1c = Boey 3 & 1,0 & 38,3 & 0,006 & $0,839 / 0,978$ \\
\hline
\end{tabular}

ABC: Área Bajo la Curva. OR: Odds rates. El rendimiento de la puntuación de Boey se demuestra mediante excelentes Odds Rates en pacientes con perforación de larga data. Combinaciones de las variables inespecíficas de esta puntuación como la patología asociada y el choque preoperatorio con la variable específica perforación mayor a 24 h, incrementan los Odds Rates.

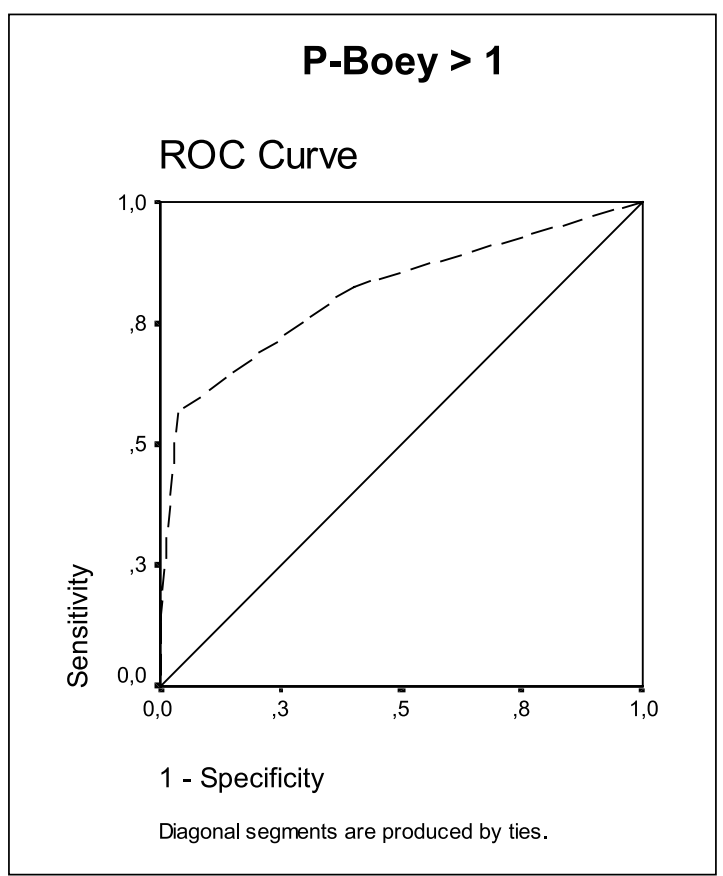

Figura 3. El ABC para P-Boey mayor a 1 fue 0,8 y es comparable al ABC para el P-POSSUM quirúrgico y predictivo.

\section{Discusión}

La evaluación de los riesgos potenciales de morbilidad y mortalidad perioperatoria con puntuaciones específicamente diseñadas para ello, es importante en la práctica clínica ${ }^{25}$. Estas puntuaciones intentan cuantificar el riesgo de morbilidad y mortalidad del paciente ${ }^{25,26}$. Se han descrito tres tipos de pun- tuaciones pronósticas para pacientes quirúrgicos ${ }^{27}$; puntaciones generales como P-POSSUM, puntuaciones específicas para morbilidad asociada como el índice cardiológico de Goodman y puntuaciones específicas para patología quirúrgica como P-Boey. Mediante el uso de puntuaciones pronósticas en pacientes sometidos a cirugía de urgencia, el cirujano tendría el respaldo para comunicar el riesgo asociado a los pacientes y familiares, además de solicitar el manejo postoperatorio en la Unidad de Paciente Crítico o en casos extremos declinar la cirugía en pacientes moribundos ${ }^{25}$.

Los factores de riesgo asociados a morbilidad y mortalidad postoperatoria en pacientes con UPP han sido extensamente estudiadas ${ }^{1-18}$. Las más importantes e influyentes son el tiempo de perforación prolongado, choque preoperatorio, edad avanzada y patología asociada ${ }^{1-19,23,24}$. Otros han demostrado que la patología asociada en estos pacientes no se asocia a complicaciones postoperatorias ${ }^{28}$. El riesgo de mortalidad después de cirugía de urgencia por UPP es $30 \%$, en pacientes ancianos con patología asociada severa este riesgo se incrementa hasta $50 \%{ }^{3,8}$. En el caso de pacientes jóvenes y saludables que se presentan precozmente el pronóstico es excelente ${ }^{3,6,8,9-14}$. La presentación y el tratamiento tardío son las principales razones para el aumento de la morbilidad y mortalidad. De acuerdo con Boey, la mortalidad llega hasta $100 \%$ de los pacientes mayores de 70 años con patología asociada que se presentan después de $24 \mathrm{~h}$ con choque preoperatorio ${ }^{3-6}$.

\section{PCR y RL}

Estos marcadores inflamatorios inespecíficos se miden frecuentemente en los servicios de urgencia. La PCR presentó un ABC moderada con un buen OR, el RL obtuvo buenos ABC y OR. El RL es me- 
dido en P-POSSUM pero la PCR no se incluye en ninguna puntuación. Probablemente, la PCR tendría que encontrar un lugar en alguna puntuación específica para UPP.

\section{Portsmouth-POSSUM}

El P-POSSUM fisiológico ha sido previamente evaluado en UPP ${ }^{19}$. Los autores encontraron una correlación importante entre una puntuación elevada y diversas complicaciones. En el presente estudio el P-POSSUM fisiológico tuvo una ABC moderada con un buen OR.

\section{Puntuación de Boey}

El análisis de P-Boey comparado con P-POSSUM, demuestra que esta simple puntuación tiene un mejor rendimiento. Esto se debe a las variables que se evalúan en cada puntuación. Cuando la variable positiva fue patología asociada, P-Boey tuvo unos $\mathrm{ABC}$ y OR pobres. Cuando la variable positiva fue choque preoperatorio el $\mathrm{ABC}$ fue bueno pero el OR fue pobre. Estos hallazgos se explican porque estas dos variables son inespecíficas del problema. Respecto al choque preoperatorio este no se asoció al evento. La variable más específica y sensible con el mejor rendimiento fue el tiempo de perforación, este tuvo excelentes $\mathrm{ABC}$ y OR porque se encuentra intrínsecamente relacionada con UPP. Así también, el tiempo de perforación tienen mejor rendimiento que cualquier parte del P-POSSUM individualmente o en conjunto. Las variables de P-Boey fueron evaluadas en todas sus posibles combinaciones y se encontró que la patología asociada junto con el choque preoperatorio, tuvieron una buena $\mathrm{ABC}$ y un pobre $\mathrm{OR}$, esto se debe al pobre rendimiento de la variable patología asociada el cual afecta negativamente al rendimiento del choque preoperatorio. Cuando las variables positivas fueron patología asociada y tiempo de perforación el $\mathrm{ABC}$ fue moderado con un buen OR, esto se explica por el efecto positivo del tiempo de perforación sobre la otra variable. Cuando las variables positivas fueron choque preoperatorio y tiempo de perforación, el $A B C$ fue bueno con un elevado OR, esto se explica por el efecto adicional de la variable inespecífica choque preoperatorio sobre la variable específica tiempo de perforación. El efecto más equilibrado se observó cuando las tres variables estuvieron presentes, es esos casos el $\mathrm{ABC}$ y el OR fueron excelentes.

El rendimiento de P-Boey fue mejor que el rendimiento de P-POSSUM debido a la variable específica tiempo de perforación. El efecto del tiempo de perforación en pacientes con UPP ha sido demostrado. La morbilidad y mortalidad postoperatoria se incrementa con el retraso en el diagnóstico y tratamiento por más de $12 \mathrm{a} 24 \mathrm{~h}$, . La razón por la cual el retraso de $12 \mathrm{~h}$ en el diagnóstico incrementa la morbilidad, es el hecho de que después de 12 $\mathrm{h}$ las perforaciones gastroduodenales se infectan, por lo que pacientes operados durante las primeras $12 \mathrm{~h}$ tendrán mejores resultados ${ }^{5}$. La morbilidad postoperatoria es considerable e incluye neumonía, infecciones de herida, abscesos intra-abdominales y complicaciones metabólicas y cardiovasculares ${ }^{3,6}$. La mortalidad postoperatoria se debe a sepsis intraabdominal, sepsis respiratoria y complicaciones cardiovasculares ${ }^{6}$.

En la práctica clínica, la diferencia entre P-Boey y P-POSSUM es que la primera se mide en el preoperatorio y su utilidad debe dirigirse a cuantificar el riesgo potencial del procedimiento quirúrgico. El P-POSSUM al medirse en el pre y postoperatorio tiene el rol de determinar la unidad más adecuada para el manejo posoperatorio del paciente.

\section{Recomendaciones para el manejo inicial}

El manejo inicial conservador de pacientes con UPP, incluyendo ensayo clínicos aleatorios, ha sido reportado ${ }^{29-33}$. La base del tratamiento conservador se encuentra en el uso de resucitación intensiva, sonda nasogástrica, antibióticos endovenosos de amplio espectro e inhibidores de la bomba de protones. Se ha reportado un elevado rango de éxito (72\%$97 \%)^{29,31,33}$, con una baja mortalidad $(0-5 \%)^{29-33}$. Al comparar la mortalidad del manejo conservador con la mortalidad de la cirugía de urgencia, el abordaje conservador parece ser más seguro por presentar menor mortalidad ${ }^{31}$. Sin embargo, ninguno de estos estudios utilizó puntuaciones como la de Boey o P-POSSUM fisiológico para definir el manejo conservador de los pacientes. De acuerdo con los resultados del presente estudio, aquellos pacientes que se presentan precozmente con una puntuación de Boey 1 ó 0, un P-POSSUM fisiológico menor a 14, una PCR menor a $37,5 \mathrm{mg} / 1$ o un RL menor a $11.600 \mathrm{~mm}^{3}$ podrían ser sometidos a un período de observación inicial.

\section{Conclusiones}

En esta serie, P-Boey tuvo un mejor rendimiento que P-POSSUM, por lo que permanece como una puntuación más específica para la evaluación de pacientes sometidos a cirugía de urgencia por UPP.

\section{Referencias}

1. Blomgern LG. Perforated peptic ulcer: Long-term results after simple closure in the elderly. World J Surg. 1997;21:412-5. 
2. Boey J, Wong J. Perforated duodenal ulcers. World J Surg. 1987;11:319-24.

3. Kujath P, Schwandner O, Bruch HP. Morbidity and mortality of perforated peptic gastroduodenal ulcer following emergency Surgery. Langenbecks Arch Surg. 2002;387:298-302.

4. Svanes C. Trends in perforated peptic ulcer: Incidence, etiology, treatment, and prognosis. World J Surg. 2000;24:277-83.

5. Svanes C, Lie RT, Svanes K, Lie SA, Søreide O. Adverse effects of delayed treatment for perforated peptic ulcer. Ann Surg. 1994;220:168-75.

6. Noguiera C, Silva AS, Santos JN, Gomes A, Ferreira J, Matos E, et al. Perforated peptic ulcer: Main factors of morbidity and mortality. World J Surg. 2003;27:782-7.

7. Testini M, Postincasa P, Piccini G, Lissidini G, Pellegrini F, Greco L. Significant factors associated with fatal outcome in emergency open Surgery for perforated peptic ulcer. World J Gastroenterology 2003;9:2338-40.

8. Rahman MM, Islam MS, Flora S, Akhter SF, Hossain S, Karim F. Mortality in perforated peptic ulcer patients after selective management of stratified poor risk cases. World J Surg. 2007;31:2341-4.

9. Boey J, Wong J, Ong GB. A prospective study on operative risk factors in perforated duodenal ulcers. Ann Surg. 1982;195:265-9.

10. Boey J, Lee NW, Koo J, Lam PH, Wong J, Ong GB. Immediate definitive surgery for perforated duodenal ulcers. Ann Surg. 1982;196:338-42.

11. Boey J, Choi SK, Alagaratnam TT, Poon A. Risk stratification in perforated duodenal ulcers. Ann Surg. 1987;205:22-6.

12. Boey J, Branicki FJ, Alagaratnam TT, Fok PJ, Choi SK, Poon A, et al. Proximal gastric vagotomy: The preferred operation for perforations in acute duodenal ulcer. Ann Surg. 1988;208:169-74.

13. Lee FY, Leung KL, Lai BS, Man SS, Dexter S, Lau WY. Predicting mortality and morbidity of patients operated on for perforated peptic ulcers. Arch Surg. 2001;136:903.

14. Lunevicius R, Morkevicius M. Risk factors influencing the early outcome results after laparoscopic repair of perforated duodenal ulcer and their predictive value. Langenbecks Arch Surg. 2005;390:413-20.

15. Rix TE, Bates T. Pre-operative risk scores for the prediction of outcome in elderly people who require emergency surgery. World J Emerg Surg. 2007;2:16.

16. Lohsiriwat V, Prapasrivorakul S, Lohsiriwat D. Perforated peptic ulcer: Clinical presentation, surgical outcomes, and the accuracy of the Boey scoring System in predicting postoperative morbidity and mortality. World J Surg. 2009;33:80-5.

17. Thorsen K, Glomsaker TB, von Meer A, Søreide K, Søreide JA. Trends in diagnosis and surgical treatment of patients with perforated peptic ulcer. J Gastrointest Surg. 2011;15:1329-35.

18. Lo HC, Wu SC, Huang HC, Yeh CC, Huang JC, Hsieh $\mathrm{CH}$. Laparoscopic simple closure alone is adequate for low risk patients with perforated peptic ulcer. World J Surg. 2011;35:1873-8.

19. Egberts JH, Summa B, Schulz U, Schafmayer C, Hinz $\mathrm{S}$, Tepl J. Impact of preoperative physiological risk profile on postoperative morbidity and mortality after emergency operation for complicated peptic ulcer disease. World J Surg. 2007;31:1449-57.

20. Copeland GP, Jones D, Walters M. POSSUM: A scoring System for surgical audit. Br J Surg. 1991;78:355-60.

21. Prytherch DR, Whiteley MS, Higgins B, Weaver PC, Prout WG, Powell SJ. POSSUM and Portsmouth POSSUM for predicting mortality. Physiological and Operative Severity Score for the enUmeration of Mortality and morbidity. Br J Surg. 1998;85:1217-220.

22. Dindo D, Demartines N, Clavien PA. Classification of surgical complications. Ann Surg. 2004; 240:205-13.

23. Koç M, Yoldaş Ö, Kiliç YA, Göçmen E, Ertan T, Dizen $\mathrm{H}$, et al. Comparison and validation of scoring systems in a cohort of patients treated for perforated peptic ulcer. Langenbecks Arch Surg. 2007;392:581-5.

24. Hanley JA, McNeil BJ. The meaning and the use of the area under a receiver operating characteristics (ROC) curve. Radiology 1982;143:29-36.

25. Chandra A, Mangam S, Marzouk D. A review of risk scoring systems utilized in patients undergoing gastrointestinal surgery. J Gastrointest Surg. 2009;13:1529-38.

26. Jones HJ, de Cossart L. Risk scoring in surgical patients. Br J Surg. 1999;86:149-57.

27. Galland RB. Severity scores in surgery: What for and who needs them? Langenbecks Arch Surg. 2002;387:5962.

28. Kim JM, Jeong SH, Lee YJ, Park ST, Choi SK, Hong $\mathrm{SC}$, et al. Analysis of risk factors for postoperative morbidity in perforated peptic ulcer. J Gastric Cancer 2012;12:26-35.

29. Donovan AJ, Vinson TL, Maulsby GO, Gewin JR. Selective treatment of duodenal ulcer with perforation. Ann Surg. 1979;189:627-36.

30. Crofts TJ, Park KG, Steele RJ, Chung SS, Li AK. A randomized trial of nonoperative treatment for perforated peptic ulcer. N Engl J Med. 1989;320:970-3.

31. Berne TV, Donovan AJ. Nonoperative treatment of perforated duodenal ulcer. Arch Surg. 1989;124:830-2.

32. Koyama Y, Hayashi T, Fujita N, Kaneko K, Takano Y, Sato N, et al. Nonoperative treatment of reperforated duodenal ulcer: Report of three cases. Surg Today 2000;30:1034-6.

33. Dascalescu C, Andriescu L, Bulat C, Danila R, Dodu L, Acornicesei M, et al. Taylor's method: A therapeutic alternative for perforated gastroduodenal ulcer. Hepatogastroenterology 2006;53:543-6. 